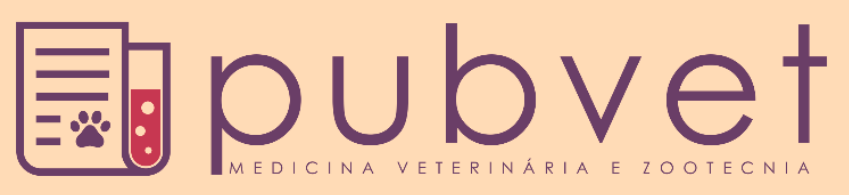

https://doi.org/10.31533/pubvet.v15n06a843.1-6

\title{
Avaliação cicatricial em cadelas submetidas à mastectomia unilateral com o uso de Tartarato de Ketanserina e Asiaticosídeo
}

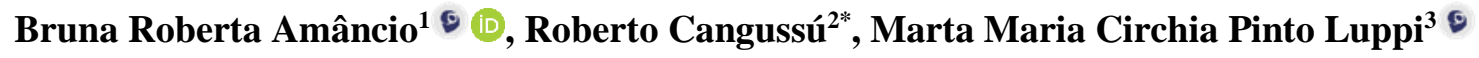 \\ ${ }^{I}$ Médica Veterinária, Mestranda em Produção Animal Sustentável pelo Instituto de Zootecnia APTA/Sertãozinho, Brasil. \\ ${ }^{2}$ Graduando em Medicina Veterinária, Hospital Veterinário da Faculdade Anhanguera, Brasil \\ ${ }^{3}$ Dra. Profa. Médica Veterinária. Faculdade Anhanguera, Brasil \\ *Autor para correspondência,E-mail: robertocangussu@hotmail.com
}

\begin{abstract}
Resumo. As neoplasias mamárias vêm sendo cada vez mais comuns em cadelas. Por ser uma doença agressiva o tratamento de eleição é a retirada cirúrgica da neoplasia juntamente com a mama acometida e adjacentes, e em determinados casos, retirada da cadeia mamária inteira. O procedimento cirúrgico intitulado mastectomia é invasivo e em determinados casos de grande extensão estando associada a maior stress cirúrgico, dor e maior incidência de complicações pós-operatórias como inflamação do tecido e deiscência de pontos. $\mathrm{O}$ objetivo do trabalho com o uso do Regepil ${ }^{\circledR}$ (Ouro Fino Saúde Animal) foi reduzir tempo de cicatrização e consequentemente reduzir os dias de retirada dos pontos. No estudo foram utilizados 10 cães de diferentes raças e idades, todas fêmeas com diagnóstico de neoplasia mamária confirmada por avaliação clínica e exame complementar. Os animais foram divididos em dois grupos, sendo um com o uso do Regepil ${ }^{\circledR}$, e outro sem o uso. O Regepil ${ }^{\circledR}$ é um cicatrizante à base de Ketanserina e asiatícosideos. A Ketanserina é um antagonista de receptores de serotonina que promove o aumento na microvascularização, proporcionando maior efetividade da cicatrização na fase inicial. O asiaticosídeo é um composto a base de Centella asiática, que age estimulando a síntese de colágeno tipo I e a maturação de rede de colágeno, promovendo maior rapidez no processo de cicatrização. $\mathrm{O}$ uso do Tartarato de Ketanserina e Asiaticosídeo nas cadelas submetidas à mastectomia unilateral mostrou-se eficaz no processo de cicatrização das feridas cirúrgicas.
\end{abstract}

Palavras-chave: Glândula mamária, mastectomia, neoplasia, patologia

\section{Cicatricial evaluation in bitches submitted to unilateral mastectomy with the use of KetanSerin and Asiaticoside Tarter}

\footnotetext{
Abstract. Mammary neoplasms have been common in bitches because it is an aggressive disease the treatment of election is the surgical withdrawal of neoplasia along with the affected and adjacent breast, and in certain cases withdrawn from the whole mammary chain. The surgical procedure titled mastectomy is invasive and in certain cases of a large extent being associated with increased surgical stress, pain and greater incidence of postoperative complications such as inflammation of the tissue and deiscence of points. The objective of working with the use of Regepil® (fine gold animal health) was to reduce healing time consequently to reduce the days of withdrawal of the points. In the study, 10 dogs of different races and ages were used, all females with a diagnosis of mammary neoplasia confirmed by clinical evaluation and complementary examination. The animals were divided into two groups, one with the use of the regepil@, and another without use. Regepil ${ }^{\circledR}$ is a ketanserine and asiaatoside-based healing. Ketanserine is an antagonist of serotonin receptors that promotes the increase in microvascularization, providing greater effectiveness of healing in the initial phase. Asiaticoside is a compound of Asian Centella,
} 
which acts by stimulating the synthesis of type I collagen and the maturity of collagen network, promoting higher in the healing process. The use of the Ketanserine and Asiaticoside tartrate in the bitches submitted to unilateral mastectomy was effective in the healing process of surgical wounds.

Keywords: Mammary gland, mastectomy, neoplasia, pathology

\section{Introdução}

O estudo das neoplasias mamárias em cadelas vem ganhando atenção nos últimos anos devido a sua alta incidência, principalmente em razão do aumento da expectativa de vida desses animais (Silva, 2006).

A maioria dos cães desenvolvem cinco pares de glândulas mamárias (Schafhäuser Júnior, 2006). No entanto, podem variar entre quatro ou seis pares, que estão dispostas em duas cadeias bilaterais simétricas que vão da região do tórax até a inguinal e localizam-se paralelamente a linha Alba. As mamas são denominadas do sentido cranial para caudal sendo: glândula torácica cranial (M1) e torácica caudal (M2), glândulas abdominais craniais (M3) e abdominais caudais (M4) e o quinto par é denominado como glândulas mamárias inguinais (M5). A mama é uma glândula sudorípara apócrina modificada, exclusivamente presente em mamíferos, formada por uma rede de ductos rodeados por estroma fibrovascular, rico em adipócitos, e a última etapa do desenvolvimento mamário ocorre na gestação (Nelson \& Couto, 2015; Neves, 2018).

O tratamento de eleição no caso de confirmação da neoplasia é a retirada cirúrgica da mama acometida. Esse procedimento chamado de mastectomia, podendo ser uni ou bi lateral, parcial, regional ou total. Nas mastectomias tanto uni ou bilateral total compreende a remoção de todas as mamas da mesma cadeia e os linfonodos correspondentes (Silva, 2006). A ressecção em bloco envolve a exérese da mama neoplásica, as mamas com as quais possui conexões linfáticas e os linfonodos correspondentes, cranial a M1 linfonodo axilar caudal a M5 linfonodo inguinal, pois há grande chance de metástase para essas regiões (Silva, 2006). Esse procedimento por ser invasivo e de grande extensão está associado à uma maior duração cirúrgica, maior stress cirúrgico, dor e maior incidência de complicações pósoperatórias e consequentemente precisa de cuidados para uma boa cicatrização, pois a tensão colocada no tecido tegumentar quando se trata de mastectomia unilateral total é grande, podendo ocorrer deiscência de pontos e inflamação exacerbada. Quando necessário deve-se fazer uso de antiinflamatórios para controle da mesma, impedindo que o ciclo-oxigenase sintetize os mediadores da inflamação através do ácido araquidônico (Oliveira \& Dias, 2012).

$\mathrm{O}$ processo de cicatrização pode ser dividido em duas etapas, regeneração e cicatrização propriamente dita, consistem em uma reação inflamatória originada no próprio organismo com o intuito de defesa frente a uma lesão celular, sendo ela causada tanto por agentes físicos, químicos ou biológicos (infecção). O processo inflamatório tem como função promover a reparação celular, sendo observados sinais como: dor, calor, rubor, tumefação e perda de função. Esses sinais estão relacionados ao aumento do fluxo sanguíneo local e aumento da permeabilidade vascular, sendo controlados por mediadores inflamatórios tais como: prostaglandinas, tromboxanos, prostaciclinas, entre outros. Este processo e desencadeado por leucócitos e plaquetas ativados, do metabolismo do ácido araquidônicoprostaglandinas e leucotrienos, das cascatas da coagulação e do complemento direcionados até o foco da lesão (Oliveira \& Dias, 2012).

O objetivo do presente estudo é avaliar a eficácia do Regepil ${ }^{\circledR}$ (Ouro Fino Saúde Animal Ltda.) no processo de cicatrização das feridas cirúrgicas de mastectomias em cadelas com tumores de mama.

\section{Relato de caso}

No presente estudo foram utilizados 10 cães de diferentes raças e idades, todas fêmeas (Tabela 1) com diagnóstico de neoplasia mamária confirmada por avaliação clínica e exame complementar.

Os animais foram divididos em dois grupos, sendo um com o uso do Regepil ${ }^{\circledR}$, com o objetivo de uma redução no tempo de cicatrização consequentemente reduzindo os dias de retirada dos pontos. A instituição preconiza retirar entre 10 e 14 dias. Outro grupo sem o uso. No grupo com o uso do Regepil ${ }^{\circledR}$ foi prescrito após a retirada do curativo o uso tópico duas vezes ao dia até a retirada dos pontos. A 
retirada do curativo foi recomendada no terceiro dia após cirurgia. Foram prescritos analgésicos e antiinflamatórios, para todos os animais, tanto para o grupo com o uso do Regepil ${ }^{\circledR}$ como para o grupo sem o uso, o anti-inflamatório foi prescrito com o intuito de prolongar a analgesia, pois os analgésicos têm um pico de ação de curta duração e por se tratar de uma cirurgia com alto índice de dor.

Tabela 1. Idade, raça e dias da retirada de pontos, dos animais submetidos à mastectomia unilateral total ou parcial com o uso ou não do Regepil ${ }^{\circledR}$

\begin{tabular}{lccccc}
\hline Animal & Idade, anos & Sexo & Raça & Uso & Retirada dos pontos, dias \\
\hline Meg & 13 & Fêmea & Poodle & Sim & 9 \\
Mel & 11 & Fêmea & SRD & Sim & 9 \\
Pit & 8 & Fêmea & Basset hound & Sim & 9 \\
Coda & 14 & Fêmea & SRD & Sim & 23 \\
Isabel & 12 & Fêmea & Pastor Alemão & Sim & 20 \\
Lola & 12 & Fêmea & SRD & Não & 15 \\
July & 10 & Fêmea & SRD & Não & 11 \\
Baby & 13 & Fêmea & SRD & Não & 12 \\
Nina & 12 & Fêmea & SRD & Não & 13 \\
Malu & 14 & Fêmea & Shihtzu & Não & 13 \\
\hline
\end{tabular}

No grupo com Regepil ${ }^{\circledR}$, alguns animais necessitaram fazer uso de mais medicamentos como a paciente Coda que o tutor não realizou as recomendações clínicas e pós-operatórias, sendo necessário o uso de antibiótico após deiscência de pontos, a paciente Isabel foi recomendado o uso de corticoide e antibiótico devido a uma congestão pulmonar ocorrida no transoperatório. As feridas foram avaliadas diariamente mediante fotos enviadas pelos tutores, e foi observado que as feridas não apresentavam hematomas, deposição de fibrina ou exsudado.

No grupo sem Regepil ${ }^{\circledR}$ as pacientes Nina e Lola, fizeram o uso de antibiótico devido deiscência de pontos e a paciente Baby fez uso de corticoide após cinco dias de cirurgia, pois apresentava hematomas ao redor da cicatriz e uma secreção de aspecto aquoso provável seroma. As pacientes foram avaliadas igualmente por fotos, onde foram observados, por exemplo, a presença de hematomas, deposição de fibrina, seroma e deiscência de pontos em alguns casos. Não foi possível fazer o histopatológico de todos os tumores, pois alguns tutores não autorizaram por questões financeiras. Segue abaixo tabela com sinais apresentados pelos pacientes.

Tabela 2. Tipos e frequência dos sinais clínicos dos animais submetidos à mastectomia unilateral com e sem o uso do Regepil ${ }^{\circledR}$

\begin{tabular}{|c|c|c|c|c|c|}
\hline Animais com Regepil & Deiscência & Seroma & Hematoma & Fibrina & Exsudato \\
\hline Meg & Ausente & Ausente & Ausente & Ausente & Ausente \\
\hline Mel & Ausente & Ausente & Ausente & Ausente & Ausente \\
\hline Pit & Ausente & Ausente & Ausente & Ausente & Ausente \\
\hline Coda* & Presente & Presente & Ausente & Ausente & Ausente \\
\hline Isabel & Ausente & Ausente & Ausente & Ausente & Ausente \\
\hline \multicolumn{6}{|l|}{ Animais sem Regepil } \\
\hline Lola & Presente & Presente & Ausente & Presente & Presente \\
\hline July & Ausente & Ausente & Ausente & Presente & Presente \\
\hline Baby & Ausente & Presente & Presente & Presente & Presente \\
\hline Nina & Presente & Presente & Ausente & Presente & Presente \\
\hline Malu & Ausente & Ausente & Ausente & Presente & Presente \\
\hline
\end{tabular}

*O tutor da paciente Coda, não realizou as recomendações clínicas e pós-operatórias. Os tutores foram informados sobre a realização da pesquisa e assinaram um termo de consentimento livre e esclarecido (TCLE) aceitando participar do estudo e autorizando a divulgação dos dados, nome, voz e imagem.

O Regepil ${ }^{\circledR}$ é um cicatrizante à base de Tartarato de Ketanserina e Asiaticosídeo. O Asiaticosídeo é um composto isolado da Centella asiatica, que age principalmente nos fibroblastos, estimulando a síntese de colágeno tipo I e equilibrando a maturação da rede de fibras de colágeno, promovendo uma cicatrização mais rápida e com maior suporte a força de tensão. O Tartarato de Ketanserina é um antagonista de receptores de serotonina, que atua no aumento da microvascularização e proporciona uma resposta inflamatória mais efetiva na fase inicial da cicatrização, ampliando a ativação de macrófagos para um controle maior da infecção na ferida. 
As fotos das figuras 1 e $\underline{2}$ foram dos dias quatro em que retiraram os curativos onde a incisão encontrava-se no pico do processo inflamatório 72h após a cirurgia e no dia 9 em que foi possível a retirada dos pontos de alguns pacientes.

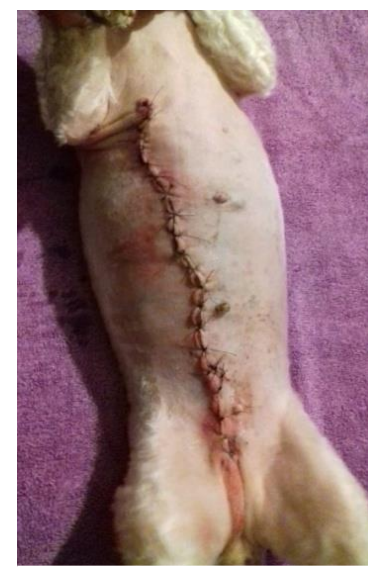

MEG DIA 04

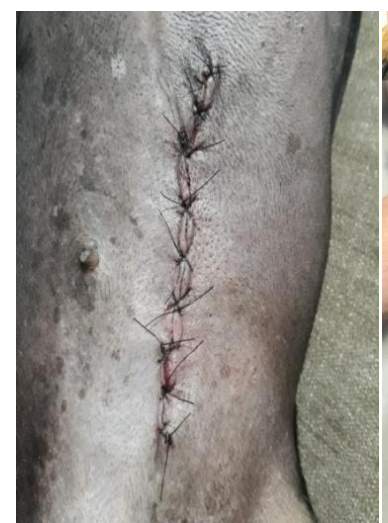

PIT DIA 04

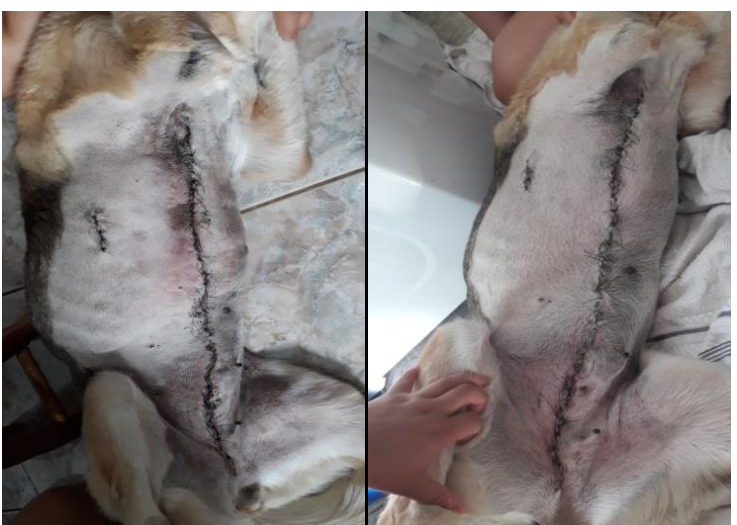

ISABEL DIA 04

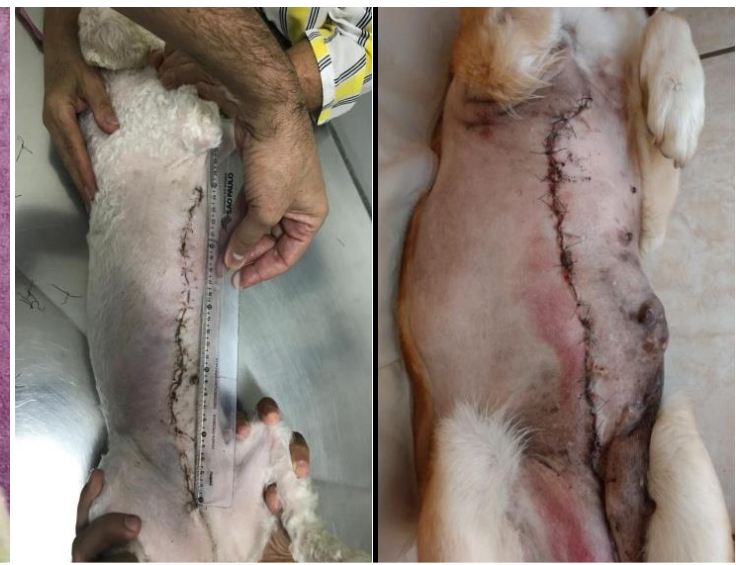

MEG DIA 09

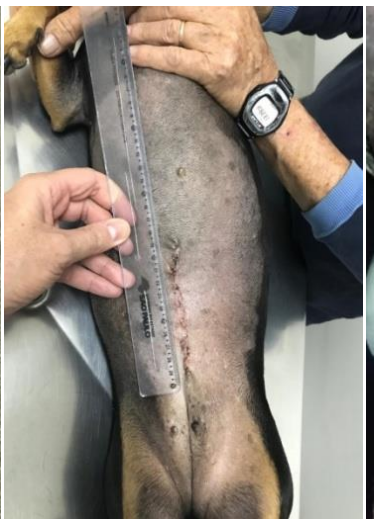

PIT DIA 09

ISABEL DIA 09

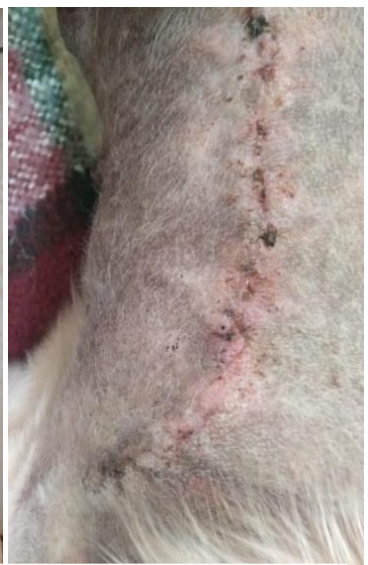

MEL DIA 09

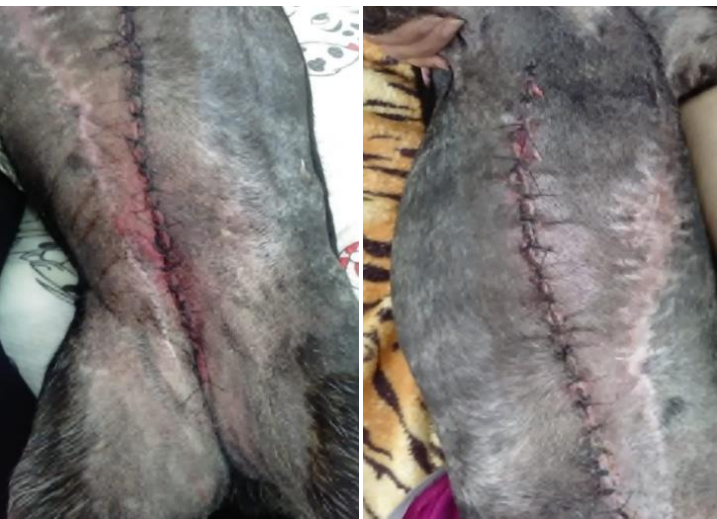

CODA DIA 04

CODA DIA 09

Figura 1. Animais que fizeram uso do Regepil@ (Ouro fino Saude Animal Ltada)

Os animais que participaram desse estudo tinham entre 08 e 14 anos. Na avaliação das fotos observou-se que entre os dias 07 e 09 era possível à retirada de todos os pontos das pacientes que usaram o Regepil ${ }^{\circledR}$, sendo menor do que no estudo de Lopes et al. (2019) onde houve a retirada de pontos com 15 dias, salvo a paciente Coda que não foram respeitadas as recomendações do pós-operatório pelo tutor e a paciente Isabel que teve os pontos retirados com 20 dias por orientação do clínico, devido ao uso do corticoide. Entretanto, mesmo com a utilização do corticoide ocorreu uma cicatrização completa como nas outras pacientes que não fizeram o uso. 


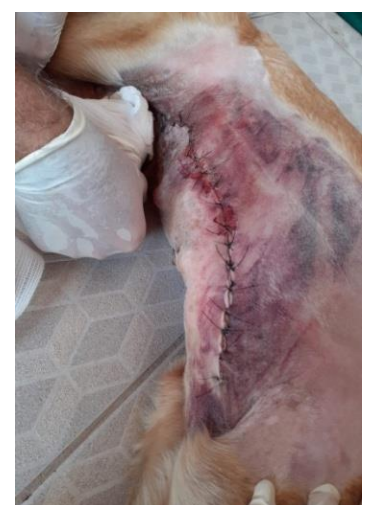

BABY DIA 04

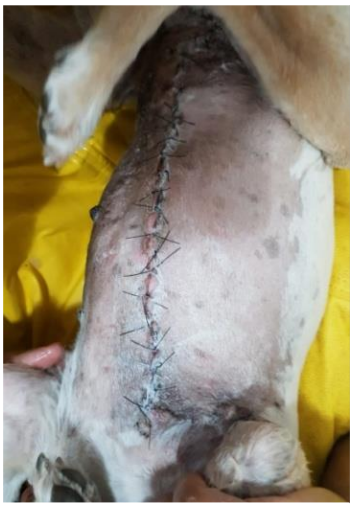

LOLA DIA 04

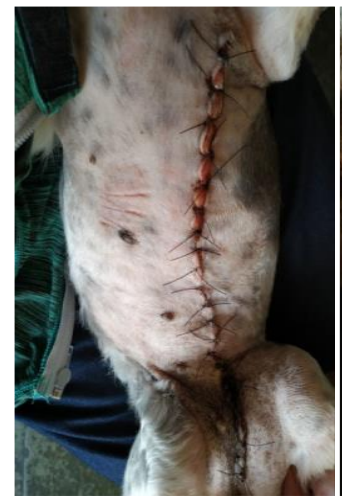

MALU DIA 04

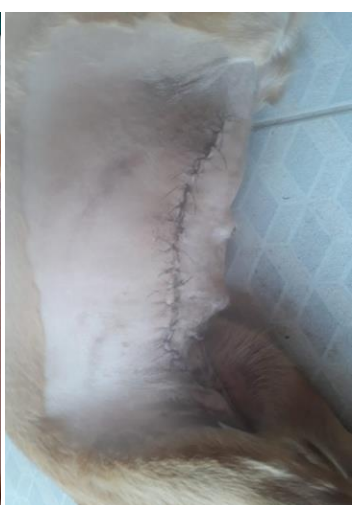

BABY DIA 09

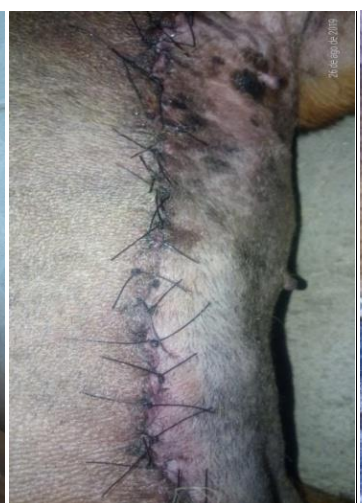

JULY DIA 04

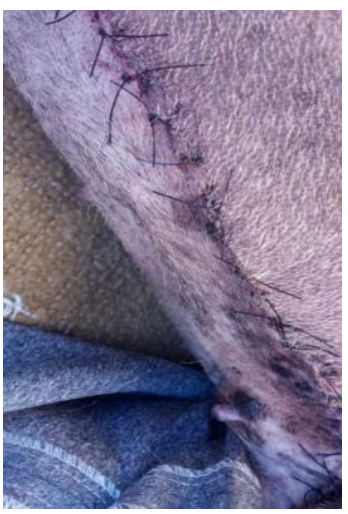

JULY DIA 09

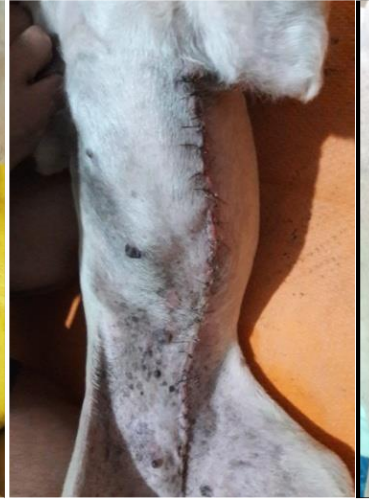

LOLA DIA 09

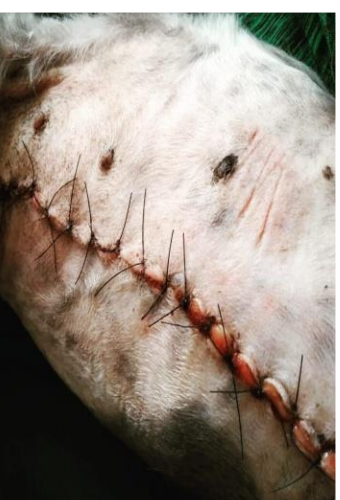

MALU DIA 09

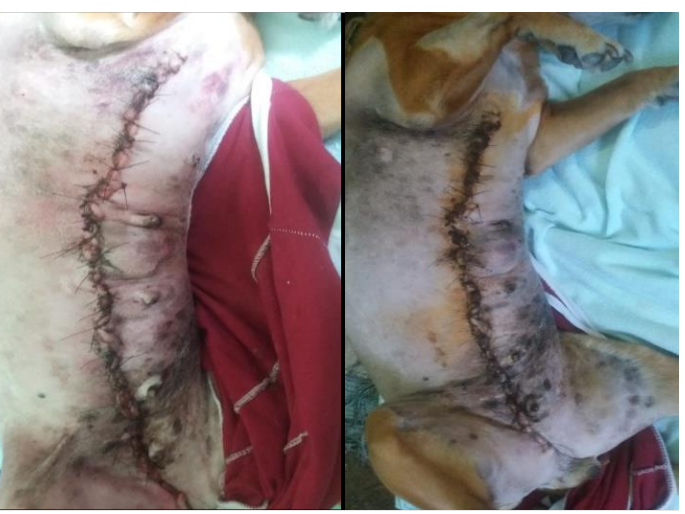

NINA DIA 04

NINA DIA 09

Figura 2. Animais que não fizeram uso do Regepil® (Ouro fino Saude Animal Ltada)

No pós-operatório foi usado somente analgésico, dipirona, tramadol e antinflamatório Maxicam comprimidos 0,5 mg (Ouro Fino Saúde Animal Ltda.), com o intuito de prolongar a ação analgésica, diferente do estudo de Lopes et al. (2019) onde se fez uso de antibiótico por 10 dias também.

\section{Conclusão}

Conclui-se que o uso do Tartarato de Ketanserina e Asiaticosídeo nas cadelas submetidas à mastectomia unilateral mostrou-se eficaz no processo de cicatrização das feridas cirúrgicas, promovendo redução do tempo de cicatrização e permitindo a retirada dos pontos antes dos protocolos já instituídos.

\section{Referências}

Lopes, R. C., Bondan, E. F., \& Stevanin, H. (2019). Estudo comparativo na cicatrização de mastectomias 
em cadelas utilizando Regepil, gentamicina e rifamicina. Revista Veterinária de Cãs e Gatos, 35(235), 68-70.

Nelson, R. W., \& Couto, C. G. (2015). Medicina interna de pequenos animais (Issue 1). Elsevier Editora.

Neves, J. M. S. R. (2018). Neoplasias mamárias em cadelas: estudo descritivo de 29 casos clínicos. Universidade Lusófona de Humanidades e Tecnologias.

Oliveira, I. V. P. M., \& Dias, R. V. C. (2012). Cicatrização de feridas: fases e fatores de influência. Acta Veterinaria Brasilica, 6(4), 267-271.

Schafhäuser Júnior, J. (2006). Desenvolvimento da glândula mamária durante a recria e sua influência no potencial produtivo de fêmeas leiteiras. Revista Da FZVA, 13(1), 128-148.

Silva, J. R. S. (2006). Mastectomia em cadelas: variações da técnica segundo a drenagem linfática da cadeia mamária-revisão de literatura. Universidade Castelo Branco, Rio de Janeiro.

Histórico do artigo

Recebido: 2 de janeiro de 2021

Aprovado: 14 de fevereiro de 2021.
Licenciamento: Este artigo é publicado na modalidade Acesso Aberto sob a licença Creative Commons Atribuição 4.0 (CC-BY 4.0), a qual permite uso irrestrito, distribuição, reprodução em qualquer meio, desde que o autor e a fonte sejam devidamente creditados. 